\title{
Avaliação da interferência do cimento endodôntico na resistência de união de um cimento resinoso à dentina
}

\author{
Evaluation of the interference of endodontic sealer in the both strength of a resin-based cement \\ Evaluación de la interferencia del sellador endodónico en resistencias del cemento a base de resina
}

Recebido: 27/05/2021 | Revisado: 06/06/2021 | Aceito: 09/06/2021 | Publicado: 23/06/2021

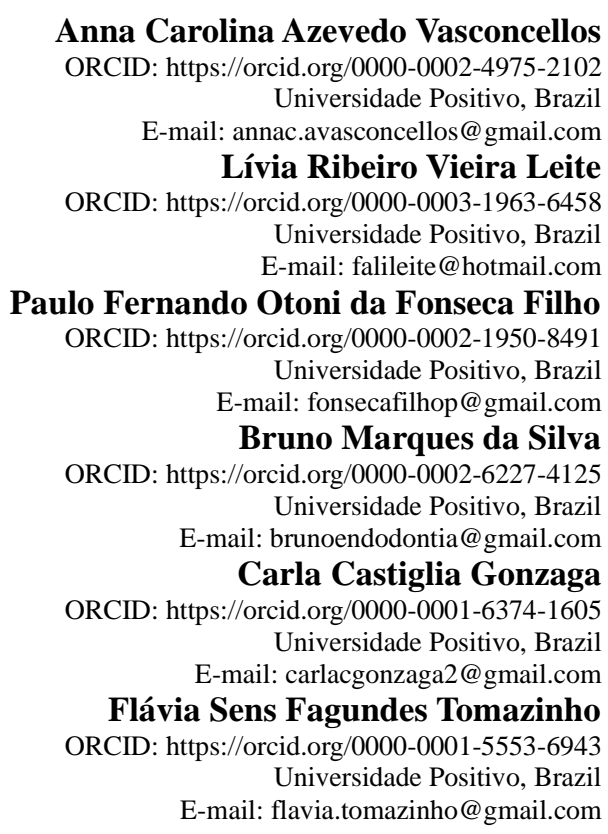

\begin{abstract}
Resumo
O objetivo desse estudo foi avaliar se o tipo e a quantidade remanescente de cimento endodôntico interferem na adesão do cimento resinoso à dentina. Para isso, foram utilizadas 25 coroas de dentes bovinos com três corpos de prova em cada coroa. Os espécimes foram divididos em 4 grupos experimentais $(n=15)$ : Grupo EF (+) - cimento Endofill com superfície parcialmente coberta de cimento endodôntico $(n=15)$, Grupo AHP $(+)-$ cimento AH Plus com superfície parcialmente coberta de cimento endodôntico $(n=15)$, Grupo EF (-) - cimento Endofill com cimento endodôntico removido em uma politriz semiautomática com disco de lixa de granulometria \#800 por 30 segundos, Grupo AHP (-) - cimento AH Plus com cimento endodôntico removido em uma politriz semiautomática com disco de lixa de granulometria \#800 por 30 segundos, e um grupo controle $(n=15)$ : Grupo $C$ - sem cimento endodôntico. Sobre a superfície de dentina foram posicionadas matrizes de silicone de cateter cirúrgico que foram preenchidas com cimento resinoso. Após a polimerização do cimento, os espécimes foram submetidos à ensaios mecânicos de resistência ao cisalhamento em uma máquina de ensaio universal. Foi registrada a força necessária para o deslocamento do corpo de prova. Os dados obtidos foram analisados pelos testes estatísticos ANOVA e Tukey com nível de significância de 5\%. Os resultados demostraram que houve diferenças estatística entre o grupo controle e os grupos experimentais, e não houve diferença estatisticamente significante entre os grupos experimentais. Pode-se concluir que a presença de cimento endodôntico interfere na adesão do cimento resinoso à dentina, independente do tipo de cimento endodôntico utilizado e da quantidade remanescente.
\end{abstract}

Palavras-chave: Cimentos de resina; Obturação do canal radicular; Resistência ao cisalhamento.

\begin{abstract}
The objective of this study was to evaluate whether the type and the remaining amount of endodontic sealer interfere with the adhesion of resin-based cement to dentin. For this, 25 crowns of bovine teeth were used with three specimens in each crown. The specimens were divided into 4 experimental groups $(n=15)$ : Group EF $(+)$ - Endofill sealer with surface partially covered with endodontic sealer $(\mathrm{n}=15)$, Group AHP $(+)$ - AH Plus sealer with surface partially covered with endodontic sealer $(n=15)$, Group EF $(-)$ - Endofill sealer with endodontic sealer removed in a semiautomatic polisher with a \# 800 sanding disc for 30 seconds, Group AHP (-) - AH Plus sealer with endodontic sealer removed in one semiautomatic polisher with sanding disc of size \# 800 for 30 seconds, and a control group ( $\mathrm{n}=$ 15): Group C - without endodontic sealer. Silicone matrices of surgical catheters were placed on the dentin surface
\end{abstract}


and filled with resin-based cement. After the polymerization of the cement, the specimens were subjected to mechanical tests of shear strength in a universal testing machine. The necessary force for the displacement of the specimen was recorded. The data obtained were analyzed by ANOVA and Tukey statistical tests with a significance level of $5 \%$. The results showed that there were statistical differences between the control group and the experimental groups, and there was no statistically significant difference between the experimental groups. It can be concluded that the presence of endodontic sealer interferes with the adhesion of resin-based cement to dentin, regardless of the type of endodontic sealer used and the remaining amount.

Keywords: Resin cements; Root canal obturation; Shear strength.

\section{Resumen}

El objetivo de este estudio fue evaluar si el tipo y la cantidad restante de sellador endodóntico interfieren con la adhesión del cemento a base de resina a la dentina. Para ello se utilizaron 25 coronas de dientes bovinos con tres ejemplares en cada corona. Las muestras se dividieron en 4 grupos experimentales $(n=15)$ : Grupo EF $(+)$ - Sellador de endodoncia con superficie parcialmente cubierta con sellador endodóntico $(n=15)$, Grupo AHP $(+)$ - Sellador AH Plus con superficie parcialmente cubierta con endodoncia sellador $(\mathrm{n}=15)$, Grupo EF $(-)$ - Sellador de endodoncia con sellador endodóntico removido en un pulidor semiautomático con un disco de lijado \# 800 durante 30 segundos, Grupo AHP (-) - Sellador AH Plus con sellador endodóntico removido en un pulidor semiautomático con disco de lijado de tamaño \# 800 durante 30 segundos, y un grupo de control $(n=15)$ : Grupo C - sin sellador endodóntico. Se colocaron matrices de silicona de catéteres quirúrgicos sobre la superficie de la dentina y se rellenaron con cemento a base de resina. Después de la polimerización del cemento, las probetas se sometieron a ensayos mecánicos de resistencia al cizallamiento en una máquina de ensayo universal. Se registró la fuerza necesaria para el desplazamiento de la muestra. Los datos obtenidos fueron analizados mediante ANOVA y pruebas estadísticas de Tukey con un nivel de significancia del 5\%. Los resultados mostraron que hubo diferencias estadísticas entre el grupo de control y los grupos experimentales, y no hubo diferencias estadísticamente significativas entre los grupos experimentales. Se puede concluir que la presencia de sellador endodóntico interfiere con la adhesión del cemento a base de resina a la dentina, independientemente del tipo de sellador endodóntico utilizado y la cantidad restante.

Palabras clave: Cementos de Resina; Obturación del conducto radicular; Resistencia al Corte.

\section{Introdução}

A restauração de dentes tratados endodonticamente pode exigir a utilização de pinos intrarradiculares para auxiliar na retenção de um núcleo ou restauração coronária. A sobrevivência da restauração final está diretamente relacionada com a retenção do pino (Baterman et al., 2003), que pode ser influenciada pela forma e design do pino (Qualtrough et al., 2003), seu comprimento e diâmetro (Aleisa et al., 2012), o tipo de agente de cimentação utilizado (Boschian-Pest et al., 2002; Kurtz et al., 2003), o preparo coronário após a cimentação (Al-Ali et al., 2003), e o cimento endodôntico (Hagge et al., 2002; Al-Ali, 2009; Cecchin et al., 2010; Lima et al., 2016).

Os cimentos endodônticos utilizados para a obturação dos canais radiculares podem influenciar na perda de retenção dos cimentos resinosos utilizados na cimentação de pinos intrarradiculares (Cecchin et al., 2010; Lima et al., 2016). Os cimentos endodônticos a base de resina epóxi tem boas propriedades físicas e biológicas (Lee et al., 2002). Os cimentos a base de hidróxido de cálcio, também tem boas propriedades físicas e biológicas e podem estimular o reparo da região apical, aumentando assim o sucesso do tratamento endodôntico (Mohammadi et al., 2014). Enquanto os cimentos a base de óxido de zinco e eugenol, são os cimentos endodônticos mais comumente utilizados por causa de sua longa história de sucesso clínico (Hagge et al., 2002), entretanto, o eugenol, como todos os fenóis, liberam radicais, que parecem inibir a polimerização da resina composta (Abo-Hamar et al., 2005; Altmann et al., 2015).

Não há consenso entre os estudos sobre se essa interação é clinicamente relevante, e não está claro se a presença de eugenol e outros componentes de cimentos endodônticos, remanescentes do tecido pulpar, e/ou a smear layer podem prejudicar a retenção dos pinos intrarradiculares (Demiryürek et al., 2010; Alesia et al., 2012; Alesia et al., 2013; Altmann et al., 2015).

A adesão às paredes de dentina de todos os cimentos resinosos depende da interface com a dentina, independentemente de isto ser ou não mediado por um sistema adesivo. A ligação adesiva-dentina depende de microrretenções criadas pela desmineralização da dentina e posterior difusão de monômeros na rede de colágeno. Estes monômeros são 
polimerizados e o embricamento micromecânico é criado (De Munck et al., 2005). Para avaliar a resistência de união do cimento resinoso com a dentina radicular, o teste de push-out é o método mais utilizado (Boschian-Pest et al. 2002, Demiryürek et al. 2010, Cecchin et al. 2011, Mosharraff \& Zare 2014, Bohrer et al. 2018).

Alguns autores, utilizando diferentes metodologias, encontraram uma redução na capacidade de retenção de pinos intrarradiculares em canais obturados com cimentos a base de óxido de zinco e eugenol (Hagge et al., 2002; Demiryürek et al., 2010; Alesia et al., 2012; Alesia et al., 2013; Bohrer et al. 2018), enquanto outros não observaram essa alteração (Aggrwal et al. 2012; Rosa et al., 2013).

Mesmo após o preparo do espaço protético, que irá receber o pino intrarradicular, pode permanecer uma camada residual de cimento endodôntico aderido a parede do canal radicular (Demiryürek et al., 2010). Essa camada pode interferir na penetração do cimento resinoso nos túbulos dentinários, podendo diminuir a resistência de união entre o pino intrarradicular/ cimento resinoso/ dentina (Alfredo et al., 2006; Vano et al., 2006).

O objetivo desse estudo foi avaliar a influência do cimento endodôntico na adesão de cimentos resinosos utilizados para a cimentação de pinos intrarradiculares. Tendo como objetivo específico, avaliar se o tipo de cimento endodôntico, resinoso ou a base de óxido de zinco e eugenol, e se a quantidade de cimento remanescente à parede de dentina interfere na força de adesão do cimento resinoso à dentina. A hipótese nula dessa pesquisa é que o cimento endodôntico e a quantidade remanescente de cimento não interferem na adesão do cimento resinoso à dentina.

\section{Metodologia}

Para este trabalho foram utilizados 25 coroas de dentes bovinos. Essas coroas foram desgastadas uma politriz semiautomática (Ecomet 3, Buehler, Lake Bluff, IL, EUA) com disco de lixa de granulometria \#300 (Tigre, Pincéis Tigre S.A., Castro, PR, Brasil) até exposição de uma superfície plana de dentina.

Após a obtenção dessas superfícies, cada coroa foi incluída em tubos de PVC com 3/4" de diâmetro com resina acrílica autopolimerizável (Dencril Industria e Comércio de Plásticos Ltda, Pirassununga, SP, Brasil), após a polimerização da resina a superfície de dentina foi regularizada em uma politriz semi-automática com disco de lixa de granulometria \#600 (Tigre, Pincéis Tigre S.A., Castro, PR, Brasil) seguido de disco de lixa de granulometria \#800 (Tigre, Pincéis Tigre S.A., Castro, PR, Brasil).

Foi aplicado EDTA 17\% por 3min. na superfície da dentina exposta para remoção da smear layer.

Depois disso, as coroas foram divididas aleatoriamente em 2 grupos experimentais $(n=10)$ e um grupo controle $(n=5)$, de acordo com o cimento endodôntico que foi utilizado: Grupo EF - Endofill (Dentsply, Petrópolis, RJ, Brasil), Grupo AHP AH Plus (Dentsply, DeTrey, Konstanz, Alemanha) e Grupo C (controle) - sem cimento endodôntico.

Os cimentos endodônticos foram manipulados seguindo as recomendações do fabricante. A superfície de dentina de cada grupo experimental foi coberta com uma fina camada de cimento endodôntico e removido o excesso com uma gaze. Os espécimes foram armazenados em estufa a $37^{\circ} \mathrm{C}$ por 24 horas em $100 \%$ de umidade.

Após 24 horas, e presa completa dos cimentos, os espécimes de cada grupo foram divididos em dois subgrupos: Subgrupo (+) - superfície parcialmente coberta de cimento endodôntico e Subgrupo (-) - superfície com cimento endodôntico removida em uma politriz semiautomática com disco de lixa de granulometria \#800 por 30 segundos.

Em seguida, 75 matrizes de silicone, foram confeccionadas a partir de cateter cirúrgico, com diâmetro de $1 \mathrm{~mm}$ e $1 \mathrm{~mm}$ de altura. Três matrizes de silicone foram posicionadas sobre cada espécime (Figura 1a) e seu volume interno foi preenchido com cimento resinoso do tipo auto-adesivo (Set PP, SDI, Bayswater, Austrália), totalizando 3 corpos de prova por espécime $(\mathrm{n}=15)$. 
O cimento foi manipulado segundo as recomendações do fabricante, inserido nas matrizes e fotoativado por 40 segundos com um aparelho fotoativador com lâmpada LED (Poly Wireless, Kavo, Joinville, SC, Brasil) com intensidade de $1100 \mathrm{~mW} / \mathrm{cm}^{2}$. Após 10 minutos, a matriz de silicone foi cuidadosamente removida com o auxílio de uma lâmina de bisturi número 15, para expor os cilindros com área de união de aproximadamente $0,79 \mathrm{~mm}^{2}$, unidos à superfície dos materiais (Figura 1b). Após a remoção das matrizes, foi realizada uma avaliação visual para verificação de possíveis defeitos. Os espécimes foram armazenados em água destilada a $37^{\circ} \mathrm{C}$ por 48 horas.

Figura 1 - (a) As matrizes de silicone posicionadas sobre cada espécime e preenchidas com cimento resinoso. (b) Após a fotopolimerização do cimento resinoso as matrizes de silicone foram cuidadosamente removidas para expor os cilindros de cimento resinoso com área de união de aproximadamente $0,79 \mathrm{~mm}^{2}$, unidos à superfície da dentina.

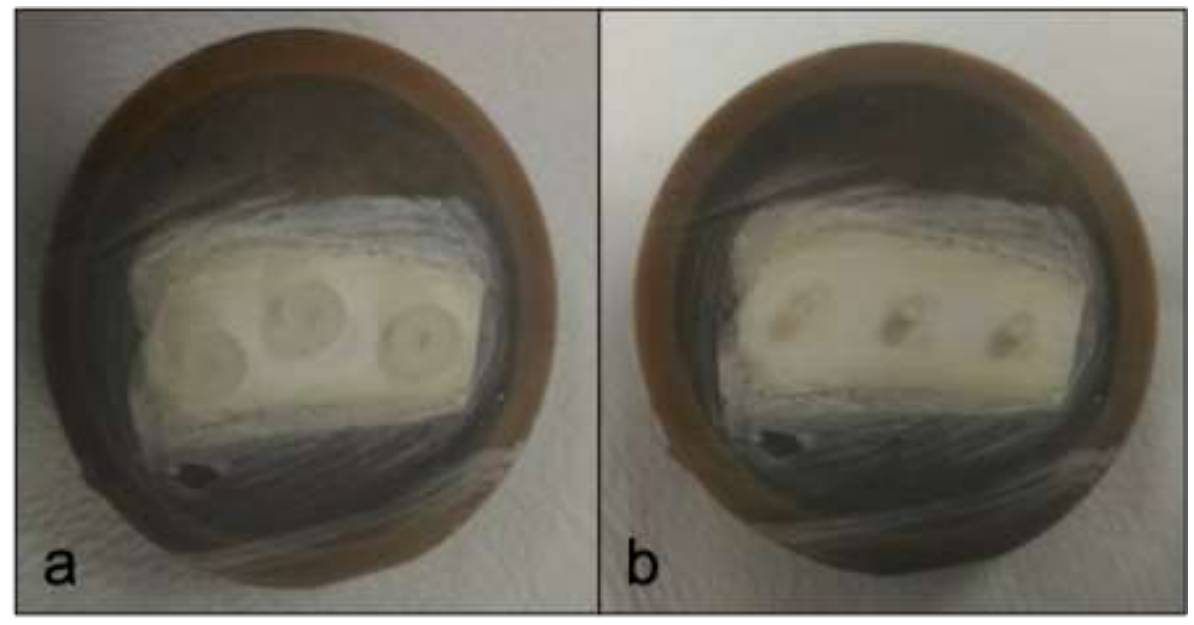

Fonte: Autores.

Decorrido o período de armazenamento, os corpos de provas foram submetidos ao ensaio mecânico de resistência ao cisalhamento em uma máquina de ensaios universal (EMIC, modelo DL2000, São José dos Pinhais, PR, Brasil), utilizando células de carga de $5 \mathrm{Kgf}$. O carregamento de cisalhamento foi aplicado na base dos cilindros com um fio de aço $(0,2 \mathrm{~mm}$ de diâmetro) com velocidade de $0,5 \mathrm{~mm} / \mathrm{min}$ até o rompimento (Figura 2). 
Figura 2 - Os corpos de provas foram submetidos ao ensaio mecânico de resistência ao cisalhamento em uma máquina de ensaios universal (EMIC, modelo DL2000, São José dos Pinhais, PR, Brasil), utilizando células de carga de 5 Kgf. O carregamento de cisalhamento foi aplicado na base dos cilindros com um fio de aço $(0,2 \mathrm{~mm}$ de diâmetro $)$ com velocidade de $0,5 \mathrm{~mm} / \mathrm{min}$ até o rompimento.

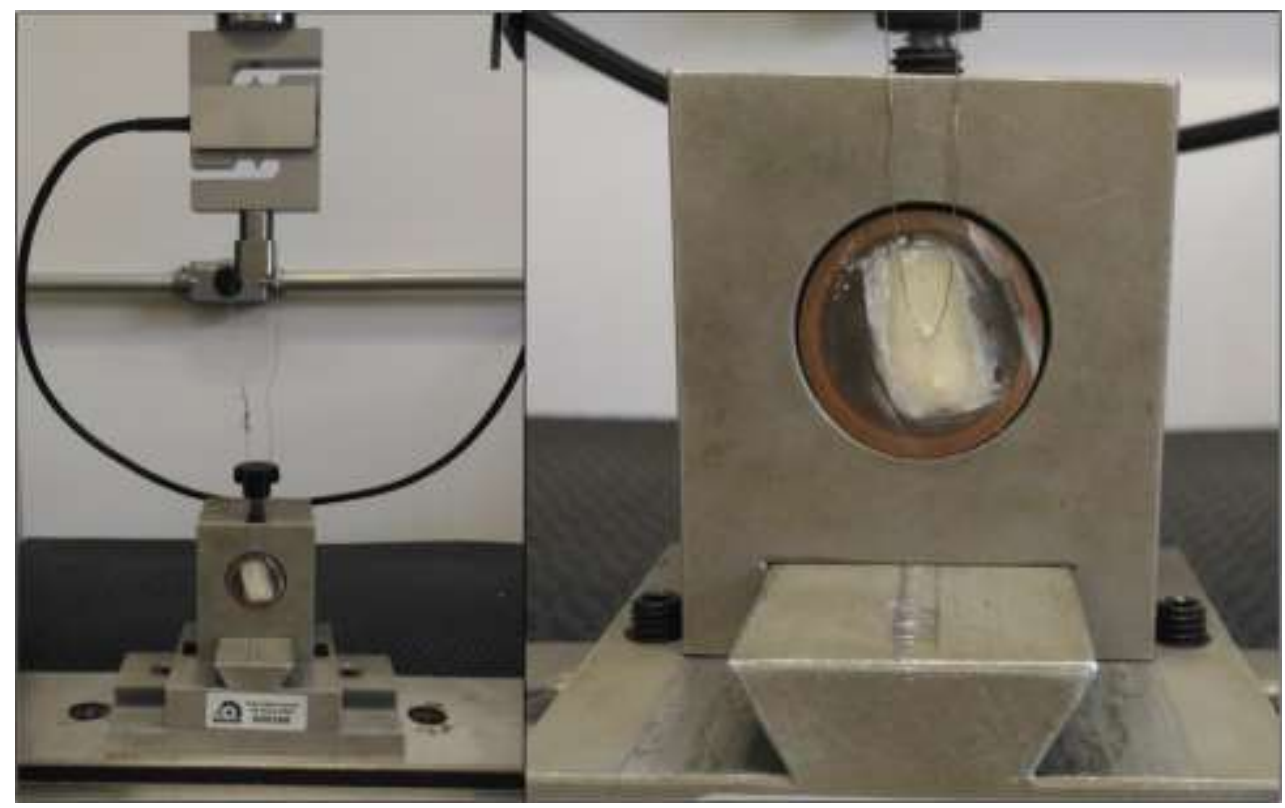

Fonte: Autores.

A resistência de união foi calculada dividindo-se a força máxima registrada durante o ensaio (em $\mathrm{N}$ ) pela área de união (em $\left.\mathrm{mm}^{2}\right)$ e expressa em Mpa.

Após a realização dos ensaios de resistência ao microcisalhamento, foi feita uma análise qualitativa das superfícies dos corpos de prova com uma lupa estereoscópica com aumento de 57x (SXZ9, Olympus, Tóquio, Japão) para determinação do tipo de falha (adesiva, coesiva ou mista).

Uma análise quantitativa dos dados foi realizada através da análise da força necessário para o deslocamento dos corpos de prova e a análise desses dados foi realizada empregando-se os testes estatísticos ANOVA e Tukey com nível de significância de $5 \%$.

\section{Resultados}

As médias e os desvios-padrão (DP) dos resultados estão expressos na Tabela 1. O teste ANOVA demonstrou que houve diferenças significativas entre os grupos $(\mathrm{p}=0,000)$. E o teste de múltipla comparação de Tukey revelou diferenças estatisticamente significativas entre os grupos experimentais e o grupo controle $(p=0,000)$. 
Tabela 1 - Média e desvio padrão (DP) da resistência de união entre o cimento resinoso e a dentina.

\begin{tabular}{ll}
\hline Grupo & Média \pm DP \\
\hline Controle & $4,26 \pm 1,63^{\mathrm{a}}$ \\
$\mathrm{EF}(+)$ & $2,03 \pm 1,50^{\mathrm{b}}$ \\
$\mathrm{EF}(-)$ & $1,63 \pm 1,26^{\mathrm{b}}$ \\
$\mathrm{AHP}(+)$ & $1,57 \pm 0,86^{\mathrm{b}}$ \\
$\mathrm{AHP}(-)$ & $0,98 \pm 0,57^{\mathrm{b}}$ \\
\hline
\end{tabular}

Nota: Letras diferentes indicam diferença estatisticamente significativa na coluna.

*ANOVA seguido de teste post hoc de Tukey $(\mathrm{p}<0,05)$.

Fonte: Autores.

A análise das superfícies dos corpos de prova para determinação do tipo de falha revelou a falha adesiva como a mais frequente, sendo observada em todos os casos, exceto em um espécime do grupo controle onde foi observada uma falha do tipo mista.

\section{Discussão}

Este estudo teve como objetivo avaliar a influência do tipo de cimento endodôntico, resinoso ou à base de óxido de zinco e eugenol, e da quantidade de cimento endodôntico remanescente à parede dentinária na adesão de cimentos resinosos utilizados para a cimentação de pinos intrarradiculares. A hipótese nula dessa pesquisa foi rejeita, uma vez que houve diferença estatística entre os grupos experimentais e o grupo controle.

Os resultados observados no presente estudo demonstraram que a presença de cimento endodôntico, independente do tipo e da quantidade remanescente, diminuiu a força de união do cimento resinoso à dentina. Esses resultados corroboram os encontrados em outros estudos (Al-Ali, 2006; Alfredo et al. 2006; Demiryürek et al., 2010; Alesia et al., 2012; Alesia et al., 2013; Altmann et al., 2015; Bohrer et al., 2018; Soares et al., 2020).

No presente estudo não houve diferença estatisticamente significante na força de união entre o cimento resinoso (Set PP) e a dentina, independente do cimento endodôntico utilizado, resinoso (AH Plus) ou a base de óxido de zinco e eugenol (Endofill). Esses resultados divergem de vários estudos que demonstraram que o Endofill diminui a força de união entre o cimento resinoso utilizado para a cimentação de pinos de fibra de vidro e a dentina (Al-Ali, 2006; Alfredo et al. 2006; Alesia et al., 2012; Alesia et al., 2013; Altmann et al., 2015; Bohrer et al., 2018).

Muitos estudos utilizam o teste de push-out para avaliar a resistência de união do cimento resinoso com a dentina radicular (Boschian-Pest et al. 2002, Demiryürek et al. 2010, Cecchin et al. 2011, Mosharraff \& Zare 2014, Bohrer et al. 2018). O teste de microcisalhamento tem como vantagens a facilidade de aplicação e validade para testes de resistência de união (Brahimi Nezhad et al., 2021); portanto, foi utilizado neste estudo para a avaliação da resistência de união entre o cimento resinoso e a dentina.

Os cimentos resinosos são utilizados para a cimentação de pinos intrarradiculares e muitas falhas ocorrem entre a parede do canal radicular e o cimento resinoso (Gomes et al., 2012). Assim, a compreensão dos fatores que interferem na união entre a parede do canal radicular e o cimento resinoso é importante (Teixeria et al., 2008). Um desses fatores de interferência é o remanescente de cimento endodôntico (Demiryürek et al., 2010).

Há informações limitadas na literatura sobre o efeito de cimentos endodônticos sobre a resistência de união dos cimentos resinosos utilizados para a cimentação de retentores intrarradiculares a dentina (Alesia et al., 2012; Alesia et al., 
2013). Na meta-análise realizada por Altmann et al. (2015) nenhum consenso foi encontrado na literatura sobre a influência de cimentos endodônticos à base de eugenol na resistência de união de pinos de fibra cimentado com cimentos resinosos em canais radiculares, nem mesmo se a influência de eugenol é diferente em diferentes processos de polimerização.

Os cimentos à base de óxido de zinco e eugenol são muito utilizados na endodontia. Estes cimentos podem liberar radicais livres que interferem na reação de polimerização de cimentos resinosos (Izadi et al., 2013). Foi observado que esse fator pode diminuir a força de união entre os pinos de fibra de vidro e a dentina do canal radicular (Al-Ali et al., 2006; Alfredo et al., 2006; Cecchin et al., 2011; Aleisa et al., 2012; Aleisa et al., 2013; Izadi et al., 2013; Mosharraff \& Zare, 2014). No entanto, outros pesquisadores relataram que os cimentos endodônticos reduzem a adesão entre os pinos de fibra de vidro e a parede do canal radicular quando utilizado um cimento resinoso, mas não foi observado diferença entre os cimentos endodônticos a base de eugenol e a base de resina (Hagge et al., 2002; Demiyürek et al., 2010). Segundo os autores, um fator importante e que pode interferir na união entre a parede do canal radicular e o cimento resinoso é o remanescente do material obturador nas paredes do canal radicular (cimento e guta-percha), independente do tipo de cimento endodôntico utilizado, e a interferência desse fator pode ser maior em canais ovalados (Hagge et al., 2002; Mosharraff \& Zare, 2014). Esses resultados corroboram os encontrado no presente estudo, uma vez que houve diferença na força de união entre à dentina e o cimento resinoso na presença de cimento endodôntico independente da quantidade de cimento endodôntico remanescente.

Os presentes achados devem ser associados a outros estudos para entender melhor influência durante a prática clínica do remanescente de cimentos endodônticos na adesão à dentina de cimentos resinosos utilizados para a cimentação de pinos de fibra de vidro.

\section{Conclusão}

Pode-se concluir que a presença de cimento endodôntico interfere na adesão do cimento resinoso à dentina, independente do tipo de cimento endodôntico utilizado e da quantidade remanescente.

\section{Referências}

Abo-Hamar, S. E., Federlin, M., Hiller, K. A., Friedl, K. H., \& Schmalz, G. (2005). Effect of temporary cements on the bond stren gth of ceramic luted to dentin. Dental Materials, 21(9), 794-803. https://doi.org/10.1016/j.dental.2005.01.015.

Aggarwal, V., Singla, M., Miglani, S., \& Kohli, S. (2012). Effect of different root canal obturating materials on push-out bond strength of a fiber dowel. Journal of Prosthodontics, 21(5), 389-392. https://doi.org/10.1111/j.1532-849X.2012.00850.x

Al-Ali K. (2009). Effect of eugenol-based root canal sealers on retention of prefabricated metal posts luted with resin cement. The Saudi Dental Journal, 21(2), 69-73. https://doi.org/10.1016/j.sdentj.2009.07.003

Al-Ali, K., Talic, Y., Abduljabbar, T., \& Omar, R. (2003). Influence of timing of coronal preparation on retention of cemented cast posts and cores. The International Journal of Prosthodontics, 16(3), 290-294.

AlEisa, K., Al-Dwairi, Z. N., Lynch, E., \& Lynch, C. D. (2013). In vitro evaluation of the effect of different endodontic sealers on retentive strength of fiber posts. Operative Dentistry, 38(5), 539-544. https://doi.org/10.2341/12-414-L

Aleisa, K., Alghabban, R., Alwazzan, K., \& Morgano, S. M. (2012). Effect of three endodontic sealers on the bond strength of prefabricated fiber posts luted with three resin cements. The Journal of Prosthetic Dentistry, 107(5), 322-326. https://doi.org/10.1016/S0022-3913(12)60084-5

Alfredo, E., de Souza, E. S., Marchesan, M. A., Paulino, S. M., Gariba-Silva, R., \& Sousa-Neto, M. D. (2006). Effect of eugenol-based endodontic cement on the adhesion of intraradicular posts. Brazilian Dental Journal, 17(2), 130-133. https://doi.org/10.1590/s0103-64402006000200009

Altmann, A. S., Leitune, V. C., \& Collares, F. M. (2015). Influence of Eugenol-based Sealers on Push-out Bond Strength of Fiber Post Luted with Resin Cement: Systematic Review and Meta-analysis. Journal of Endodontics, 41(9), 1418-1423. https://doi.org/10.1016/j.joen.2015.05.014

Bateman, G., Ricketts, D. N., \& Saunders, W. P. (2003). Fibre-based post systems: a review. British Dental Journal, 195(1), 43-37. https://doi.org/10.1038/sj.bdj.4810278

Bohrer, T. C., Fontana, P. E., Wandscher, V. F., Morari, V., Dos Santos, S. S., Valandro, L. F., \& Kaize, O. B. (2018). Endodontic Sealers Affect the Bond Strength of Fiber Posts and the Degree of Conversion of Two Resin Cements. The Journal of Adhesive Dentistry, 20(2), 165-172. https://doi.org/10.3290/j.jad.a40301 
Boschian Pest, L., Cavalli, G., Bertani, P., \& Gagliani, M. (2002). Adhesive post-endodontic restorations with fiber posts: push-out tests and SEM observations. Dental Materials, 18(8), 596-602. https://doi.org/10.1016/s0109-5641(02)00003-9

Cecchin, D., Farina, A. P., Souza, M. A., Carlini-Júnior, B., \& Ferraz, C. C. (2011). Effect of root canal sealers on bond strength of fibreglass posts cemented with self-adhesive resin cements. International endodontic journal, 44(4), 314-320. https://doi.org/10.1111/j.1365-2591.2010.01831.x

De Munck, J., Van Landuyt, K., Peumans, M., Poitevin, A., Lambrechts, P., Braem, M., \& Van Meerbeek, B. (2005). A critical review of the durability of adhesion to tooth tissue: methods and results. Journal of Dental Research, 84(2), 118-132. https://doi.org/10.1177/154405910508400204

Demiryürek, E. O., Külünk, S., Saraç, D., Yüksel, G., \& Bulucu, B. (2009). Effect of different surface treatments on the push-out bond strength of fiber post to root canal dentin. Oral Surgery, Oral Medicine, Oral Pathology, Oral Radiology, and Endodontics, 108(2), e74-e80. https://doi.org/10.1016/j.tripleo.2009.03.047

Demiryürek, E. O., Külünk, S., Yüksel, G., Saraç, D., \& Bulucu, B. (2010). Effects of three canal sealers on bond strength of a fiber post. Journal of Endodontics, 36(3), 497-501. https://doi.org/10.1016/j.joen.2009.11.014

Ebrahimi Nezhad, M., Jafarpour, D., \& Saravi, M. S. (2021). Effect of ultrasonic vibration on microshear bond strength of flowable composite to enamel. Brazilian Dental Science, 24(1), 1-6. https://doi.org/10.14295/bds.2021.v24i1.2224.

Gomes, M. F., Botta, S. B., Matos, A. B., \& Netto, N. G. (2012). The interference of the cleaning procedure of root walls with two different solvents on the adhesion of fiberglass intraradicular posts. The Journal of Contemporary Dental Practice, 13(3), 275-279. https://doi.org/10.5005/jp-journals-10024-1136

Hagge, M. S., Wong, R. D., \& Lindemuth, J. S. (2002). Effect of three root canal sealers on the retentive strength of endodontic posts luted with a resin cement. International Endodontic Journal, 35(4), 372-378. https://doi.org/10.1046/j.0143-2885.2001.00493.x

Izadi, A., Azarsina, M., \& Kasraei, S. (2013). Effect of eugenol-containing sealer and post diameter on the retention of fiber reinforced composite posts. Journal of Conservative Dentistry, 16(1), 61-64. https://doi.org/10.4103/0972-0707.105301

Kurtz, J. S., Perdigão, J., Geraldeli, S., Hodges, J. S., \& Bowles, W. R. (2003). Bond strengths of tooth-colored posts, effect of sealer, dentin adhesive, and root region. American Journal of Dentistry, 16 Spec No, 31A-36A.

Lee, K. W., Williams, M. C., Camps, J. J., \& Pashley, D. H. (2002). Adhesion of endodontic sealers to dentin and gutta-percha. Journal of Endodontics, 28(10), 684-688. https://doi.org/10.1097/00004770-200210000-00002

Lima, A. C., Rached-Junior, F. J., Faria, N. S., Messias, D. C., Chaves, C., Freitas, J. V., Baratto-Filho, F., \& Silva-Sousa, Y. T. (2016). Influence of Sealer and Light-Curing Units on Push-Out Bond Strength of Composite Resin to Weakened Roots. Brazilian Dental Journal, 27(4), 430-435. https://doi.org/10.1590/0103-6440201601010

Mohammadi, Z., Karim Soltani, M., Shalavi, S., Yazdizadeh, M., \& Jafarzadeh, M. (2014). Calcium hydroxide-based root canal sealers: an updated literature review. Compendium of Continuing Education in Dentistry, 35(5), 334-340.

Mosharraf, R., \& Zare, S. (2014). Effect of the type of endodontic sealer on the bond strength between fiber post and root wall dentin. Journal of Dentistry, 11(4), 455-463.

Qualtrough, A. J., Chandler, N. P., \& Purton, D. G. (2003). A comparison of the retention of tooth-colored posts. Quintessence International, 34(3), 199-201. Rosa, R. A., Barreto, M. S., Moraes, R., Broch, J., Bier, C. A., Só, M. V., Kaizer, O. B., \& Valandro, L. F. (2013). Influence of endodontic sealer composition and time of fiber post cementation on sealer adhesiveness to bovine root dentin. Brazilian Dental Journal, 24(3), 241-246. https://doi.org/10.1590/01036440201302154

Soares, I., Crozeta, B. M., Pereira, R. D., Silva, R. G., \& da Cruz-Filho, A. M. (2020). Influence of endodontic sealers with different chemical compositions on bond strength of the resin cement/glass fiber post junction to root dentin. Clinical Oral Investigations, 24(10), 3417-3423. https://doi.org/10.1007/s00784-020$03212-9$

Teixeira, C. S., Pasternak-Junior, B., Borges, A. H., Paulino, S. M., \& Sousa-Neto, M. D. (2008). Influence of endodontic sealers on the bond strength of carbon fiber posts. Journal of Biomedical Materials Research, 84(2), 430-435. https://doi.org/10.1002/jbm.b.30888

Vano, M., Cury, A. H., Goracci, C., Chieffi, N., Gabriele, M., Tay, F. R., \& Ferrari, M. (2006). The effect of immediate versus delayed cementation on the retention of different types of fiber post in canals obturated using a eugenol sealer. Journal of Endodontics, 32(9), 882-885. https://doi.org/10.1016/j.joen.2006.02.025 